\title{
Time to Improve and Recover from Depressive Symptoms and Interpersonal Problems in a Clinical Trial
}

\author{
Rodrigo T. Lopes, ${ }^{1}$ Miguel M. Gonçalves, ${ }^{1 *}$ Daniel Fassnacht, ${ }^{2}$ Paulo P. P. Machado ${ }^{1}$ \\ and Inês Sousa ${ }^{3}$ \\ ${ }^{1}$ School of Psychology, University of Minho, Braga, Portugal \\ ${ }^{2}$ Department of Psychology, James Cook University, Singapore, Singapore \\ ${ }^{3}$ Department of Mathematics and Applications, University of Minho, Braga, Portugal
}

\begin{abstract}
Results from an earlier clinical trial comparing narrative therapy with cognitive-behavioural therapy (Lopes et al., 2013) suggested that narrative therapy is efficacious for depression. However, there were significant differences in symptom reduction on the Beck Depression Inventory-II, favouring cognitive-behavioural therapy, if dropouts were included in the analysis, suggesting that time to recovery or improvement would differ in both treatments. Contrarily, results showed that treatment assignment was not a predictor for differential effect. Using a survival analytic approach, it was found that four sessions were necessary for $\mathbf{5 0 \%}$ improvement and 16 sessions for $50 \%$ recovery. Additionally, depressive symptoms changes occurred significantly faster than interpersonal changes, again regardless of treatment assignment. These results support previous findings of the dose-response literature and of the phase model of change, with the advantage of being specific to psychotherapy with depressive clients. Copyright $\odot 2013$ John Wiley \& Sons, Ltd.
\end{abstract}

Key Practitioner Message:

- For 50\% of clients with major depressive disorder, it takes four sessions to improve and 16 sessions to recover, regardless of whether they were treated with narrative therapy or cognitive-behavioural therapy. For those clients who recover, they do so by session 11.

- Clients change depressive symptoms more consistently and much faster than they change interpersonal problems. For clients who will not recover during brief interventions and especially for clients who present strong interpersonal problems at onset, long-term treatment plans should be considered.

- More emphasis should be laid on symptomatic relief in the early stages of treatment and on interpersonal issues at later stages.

Keywords: Depression, Treatment of Depression, Psychological Treatment of Depression, Empirically Supported Therapy (EST), Narrative Therapy, Cognitive-Behavioural Therapy, Survival Analysis

Results from an earlier controlled clinical trial comparing narrative therapy (NT) with cognitive-behavioural therapy (CBT) for depression (Lopes et al., 2013) showed significant differences in symptom reduction according to the Beck Depression Inventory-II (BDI-II; Beck, Steer, \& Brown, 1996), favouring CBT, but only on the intend-to-treat (ITT) analyses. This finding suggests that although both treatments are equally effective for those who complete treatment, the timing in which clients attain reliable improvement may differ in these treatments. Moreover, despite this difference on the BDI-II, no differences (either in the ITT and or the completer samples) were found on the Outcome

*Correspondence to: Miguel M. Gonçalves, School of Psychology, University of Minho, Braga, Portugal.

E-mail: mgoncalves@psi.uminho.pt
Questionnaire-45.2 (OQ-45.2; Lambert et al., 1996a), which measures psychological functioning more globally (besides symptoms, it also measures social functioning and interpersonal problems). This raised the question of whether treatments had differential effects on depressive symptoms and interpersonal problems. Thus, this study targets these two issues: the time needed for change in both treatments and the time needed for change in depressive symptoms and interpersonal problems also in both treatments.

Time of Improvement within Psychotherapeutic Treatment

A vast majority of evidence in psychotherapy outcome research points out a positive correlation between the 
$R$. Lopes et al.

amount of psychotherapy provided and its benefit. In an important meta-analysis, Howard, Kopta, Krause, and Orlinsky (1986) assessed 2431 outpatients. 'Dose' was defined as the number of sessions of psychotherapy the client had been exposed to, which is a common unit in across all psychotherapies. 'Effect' was defined as the percentage of clients who improved at a certain point of therapy. The results yielded that between $29 \%$ and $38 \%$ of the clients improved between the first three sessions. Then, half of the clients improved after eight sessions of therapy, and $75 \%$ of the clients would still respond to psychotherapy after 26 sessions, independently of the ultimate number of treatment sessions. One major limitation of this study is the lack of an operationalized definition of improvement, which relied on therapist and client ratings without using standardized measures of clinical change.

This limitation was addressed by other researchers (Anderson \& Lambert, 2001; Hansen \& Lambert, 2003; Hansen, Lambert, \& Forman, 2002; Harnett, O'Donovan, \& Lambert, 2010; Kadera, Lambert, \& Andrews, 1996; Kopta, Howard, Lowry, \& Beutler, 1994), who used the clinically significant change criteria (Jacobson \& Truax, 1991). According to this method, a client is considered to have 'improved' when pre-treatment to post-treatment change is statistically reliable; i.e., change at post-treatment is greater than the standard error of the outcome instrument (also referred to as the reliable change index [RCI]). In order to be considered 'recovered', the client must improve reliably, and the scores should lie within the limits of the functional population. This definition became the most used to assess clinically significant change in psychotherapy research (Bauer, Lambert, \& Nielsen, 2004; Ogles, Lunnen, \& Bonesteel, 2001).

There seems to be a consensus among psychotherapy researchers that the overall shape of the improvement trajectories reflects rapidly increasing rates in the early stages of treatment (Anderson \& Lambert, 2001; Harnett et al., 2010; Howard et al., 1986; Kadera et al., 1996), which progressively slow down as the number of sessions increases; i.e., the greater the doses, the fewer clients benefit from the treatment (Barkham et al., 1996). However, there seems to be a great discrepancy around the exact doses needed to attain a certain level of clinically significant change, which varies according to the clinical setting and client population. Generally, it has been found that $25 \%$ of clients reach clinically significant change between 2 and 8 sessions of psychotherapy, $50 \%$ of clients between 8 and 21 sessions and $75 \%$ of clients with more than 25 sessions (Anderson \& Lambert, 2001; Hansen et al., 2002; Harnett et al., 2010; Howard et al., 1986; Kadera et al., 1996; Kopta et al., 1994; Lambert, 2007). Further investigation about the number of sessions required to attain clinical improvement is thus needed to establish more accurate estimates (Kadera et al., 1996), specially within controlled experimental settings and with homogenous client samples (Anderson \& Lambert, 2001).

\section{Symptomatic versus Interpersonal Change in Psychotherapy}

Howard, Lueger, Maling and Martinovich (1993) proposed a descriptive model of change in psychotherapy, called the 'phase model'. Change is proposed to occur through the progression across a sequence of three phases that have been labelled remoralization, remediation and rehabilitation. In the remoralization phase, the client seeks to enhance subjective well-being through connecting himself or herself to the therapist who helps the client in getting a feeling of hope that the problematic experience will change. The remediation phase targets the alleviation of the client's symptoms and life problems. The rehabilitation phase starts at the point where symptomatic distress has abated, and the client focuses on the patterns of functioning that may have led to the precipitating problems and symptoms. The therapeutic work is then directed to acquire a better mode of functioning, in which, usually, the interpersonal domain is the main focus.

The phase model was empirically supported by the results indicating that remoralization preceded remediation, which in turn preceded rehabilitation (Hilsenroth, Ackerman, \& Blagys, 2001; Howard et al., 1993; Swift, Callahan, Heath, Herbert, \& Levine, 2010). Consistent with the phase model, Kopta et al. (1994) found different patterns of recovery for different clusters of symptoms, with acute distress requiring less time to recover (five sessions for $50 \%$ of the clients) than chronic distress (14 sessions for $50 \%$ of the clients). Finally, for the cluster the authors called 'characterological symptoms', which consist mainly of interpersonal problems, the required dose increases up to more than 104 sessions for $50 \%$ of the clients to recover (Barkham et al., 1996; Kopta et al., 1994).

Two other related researches attempted to study the hypothesis of differential time for improvement and offer empirical support for the phase model. Both studies included CBT treatment in the research. In the first study, Barkham et al. (1996) assessed 212 psychotherapy clients with a first diagnosis of major depressive disorder (MDD; American Psychiatric Association, 1980) using the BDI (Beck, Ward, Mendelson, Mock, \& Erbaugh, 1961) to assess depressive symptoms and the Inventory of Interpersonal Problems (IIP)-32 (Horowitz, Alden, Wiggins, \& Pincus, 2000; Horowitz, Rosenberg, Baer, Ureño, \& Villaseñor, 1988) to assess interpersonal problems. To study the doseeffect relation, clients were randomized to four experimental conditions: CBT or interpersonal therapy, plus a varying treatment length (8 versus 16 sessions). Results indicate that there were no differences between the two treatment approaches. However, the recovery rates for depressive 
symptoms versus interpersonal problems differed across different durations of treatments: clients who received more therapy (16 sessions) had a significantly higher proportion of recovery in the interpersonal problems scale than those clients who only received eight sessions. Moreover, the proportion of clients who achieved recovery on depressive symptoms was significantly lower than the proportion of clients who achieved recovery on the interpersonal level, at all tested durations of treatment.

In the second study, Barkham, Rees, Stiles, Hardy and Shapiro (2002) used a quasi-experimental design to assess 105 moderately depressed clients assigned to three conditions: a 2-, 8- and 16-sessions intervention samples. The results replicated the general trend of the dose-effect research: larger numbers of sessions were associated with a larger proportion of clients recovering. Specifically, and more importantly, the authors revealed that whereas many clients recovered from depressive symptoms in the three groups, larger proportions of clients recovered in the 8- and 16-sessions groups. Alternatively, the proportions of recovered clients according to the IIP-32 were very low in the two- and eight-sessions conditions but were increased in the 16-sessions group.

Up to date, except the earlier mentioned study by Lopes et al. (2013), the clinical trial by Vromans and Schweitzer (2011) remains the only study, to our knowledge, testing the efficacy of NT for depression by using a treatment manual and a reasonable sample size $(n=47)$. Although no direct comparison between change in depressive symptoms and interpersonal problems was presented, the authors showed stronger effect sizes (ESs) for depressive symptoms $(d=1.10)$ compared with interpersonal problems $(d=0.52)$, indicating that change in depressive symptoms was greater than change in interpersonal problems. Additionally, it was found that whereas improvements in depressive symptoms were maintained at 3-month follow-up, improvements in interpersonal symptoms were not.

Hence, the main aim of this study is twofold: (1) to investigate the time it takes for depressed clients to attain change and (2) to assess whether the courses of depressive symptoms and interpersonal problems are different, as proposed previously by Howard et al. (1993). Moreover, as we have two different treatments, we aim to study if these treatments have any differential impact both on the pace of global change and on changes in depressive symptoms, compared with interpersonal problems.

\section{METHOD}

A brief summary of procedures of the clinical trial from which these data are taken will be given. These procedures were described in more details elsewhere (Lopes et al., 2013).

\section{Clients}

Sixty-three clients (NT: $n=34, \mathrm{CBT}: n=29)$, diagnosed with MDD according to the Diagnostic and Statistical Manual of Mental Disorders, fourth edition (American Psychiatric Association, 2000), were assigned to a treatment condition, according to their incoming order-one to NT, the next to CBT and so on. Some clients (20.6\%) were included in the sample with a secondary anxiety diagnosis or symptoms. Baseline demographic and clinical characteristics were equivalent for both groups: mean age in the mid-30s $(M=35.44$, standard deviation $[S D]=11.51)$, a majority of female clients ( $81 \%$ female), coming from a middle to high social economic class and mostly single. Global Assessment of Functioning (American Psychiatric Association, 2000) was on average $60(S D=10)$, which corresponds to moderate symptoms and moderate impact on social or occupational functioning. A considerable number of clients were taking psychoactive medication at the beginning of treatment $\left(\mathrm{NT}=64.7 \%\right.$; $\mathrm{CBT}=55.2 \%, x^{2}(1)=0.59$, $p=0.44)$.

\section{Therapists}

All of the 10 therapists were psychologists and had on average 1.9 years of experience $(S D=2.13)$. Weekly supervision, using videotapes of sessions, was provided in order to assure the quality of the interventions and the adherence to the manual. Details of therapists' characteristics and training are given elsewhere (Lopes et al., 2013).

\section{Treatment Conditions}

The CBT manual used in this clinical trial was based on the typical CBT treatment manual for depression (see, e.g., Beck, Rush, Shaw, \& Emery, 1979; Fennel, 1997; Leahy \& Holland, 2000; Lopes et al., 2013; Rush, Beck, Kovacs, \& Hollon, 1977), which relies on the principle that depressive symptoms are maintained due to a dysfunctional way of interpreting reality. The client is therefore encouraged to try new ways of thinking about himself or herself, the world and/or others and to test those new reformulated hypothesis in reality. A behavioural activation module precedes the cognitive restructuring phase. Since literature about CBT for depression is vast, we refer the reader to the original treatment manual upon which our treatment was based (Beck et al., 1979; Leahy \& Holland, 2000).

Narrative therapy focuses on the role of narrative processes in the organization of experience, knowledge and behaviour. Problems arise as autobiographical narratives that are restricted to problematic contents, which White and Epston (1990) call problem-saturated narratives. The purpose of psychotherapy is to help clients shape new identities and narrate stories in richer and more gratifying 
ways. Thus, the therapist engage the client in activities to 'rewrite' self-narratives, e.g., by encouraging the externalizing conversations (White, 2007), which involve the use of language in a way that detaches (and sometimes even personifies) the problem from the person. Tasks assignments are suggested to intensify the person's awareness of the influence of the problem and the processes by which it maintains a deleterious influence on daily life, such as self-observation tasks or letters addressed to the problem, often from an externalizing perspective. The new alternative narrative elements that arise are called unique outcomes (White, 2007). The therapist helps the client to identify and elaborate unique outcomes by exploring how they emerged, what made them possible, what do they mean for the client's life or what are their implications for the life of the person (White, 2007). For an overview of the psychotherapeutic process and further details of the strategies and techniques, we refer the reader to the original unpublished manual (Gonçalves \& Bento, 2008, upon request) and to the previous works of White (2007; White \& Epston, 1990), upon which the manual was based. Neither of the clinical manuals laid special emphasis on the interpersonal area, but they were flexible enough to deal with interpersonal issues when they were evaluated to be at the core of the client's problem.

\section{Length of Treatment}

In both conditions, treatment was planned to be 20 weekly 60-min sessions. Sessions 1 to 16 were scheduled weekly, whereas sessions 17 to 20 were scheduled every other week. Overall, clients received on average 13.8 sessions $(S D=6.8)$. NT clients received an average of 12.94 sessions $(S D=7.05)$, whereas CBT clients received an average of 14.90 sessions $(S D=6.48) ; t(61)=-1.13, p=0.26$. Termination was initiated either after 20 sessions, as scheduled by the treatment manual (regardless of the clinical status of the client), or as mutually agreed between the therapist and the client at an earlier stage (in case the client has reached the therapeutic goals). Dropout was defined as the unilateral termination by the client without the therapist's approval or knowledge (Richmond, 1992) and/or failure to attend the last scheduled appointment (Hatchett, Han, \& Cooker, 2002; Hatchett \& Park, 2003). Fourteen clients (41.2\%) dropped out in the NT group compared with nine (31\%) in the CBT group; however, this difference was not statistically significant (Fisher's exact probability $=0.27$ ).

\section{Measures}

Structured Clinical Interview for Diagnostic and Statistical Manual of Mental Disorders, fourth edition

The SCID-I (First, Spitzer, Gibbons, \& Williams, 2002) is the most used diagnostic interview in research and was used to assess the presence of MDD, the existence of comorbidities, medication use at the onset, previous suicide attempts and comorbidities. Also, to assess the initial severity of participants at pre-treatment, the Global Assessment of Functioning Scale (American Psychiatric Association, 2000) was used. The SCID-II (First, Gibbon, Spitzer, Williams, \& Benjamin, 1997) was used to assess the presence of a personality disorder, which was an exclusion criterion.

\section{Beck Depression Inventory-II (Beck et al., 1996)}

The BDI-II was the primary measure of depressive symptoms. It consists of 21 self-report depressive symptoms rated on a 4-point scale ranging from 0 to 3 , and total score ranges from 0 to 63 . It has been translated and validated with a Portuguese sample (Campos \& Gonçalves, 2011; Coelho, Martins, \& Barros, 2002). In the present analyses, the BDI-II total score is considered to be an index of acute and chronic distress, as defined by Kopta et al. (1994), and its reduction an indication of remoralization and remediation, as proposed by Howard et al. (1993).

\section{Outcome Questionnaire-45.2 Interpersonal Relations (OQ-45.2 IR) subscale (Lambert et al., 1996a)}

The 11 self-report items of OQ-45.2 IR subscale were extracted from the OQ-45.2 (de Jong et al., 2007; Lambert et al., 1996a). It measures a range of interpersonal complaints such as loneliness, conflicts with others, family and marriage problems and sexual life, which are rated on a five-point Likert scale ranging from 0 to 4 . Thus, the total score ranges from 0 to 44 , where high scores indicate interpersonal problems and low scores indicate satisfaction with interpersonal relations (Lambert et al., 1996b). Lambert et al. (1996a) found good internal consistency $(\alpha=0.74)$ and testretest reliability measures $(r=0.80)$. Furthermore, Lambert et al. (1996a) indicated that the OQ-45.2 IR subscale correlates significantly with all but two subscales of the IIP (Horowitz et al., 2000), the most widely used measure of interpersonal functioning. For a comparison with the results obtained by Kopta et al. (1994) and Barkham et al. (1996), we assume that the OQ-45.2 IR subscale is equivalent to the 'characterological' symptoms, which are interpersonal in nature. In the phase model, it would reflect the rehabilitation phase (Hilsenroth et al., 2001; Howard et al., 1993; Swift et al., 2010).

Clients were asked to complete both questionnaires every fourth session (i.e., at sessions 1, 4, 8, 12, 16 and 20), which are handed to the participants before the session took place.

\section{Analysis}

\section{Definition of Clinically Significant Change}

A standardized definition of clinical change was used (Jacobson \& Truax, 1991). The values that determine the threshold for the clinical and non-clinical samples and the RCI are given below. According to norms gathered from meta-analysis (Seggar, Lambert, \& Hansen, 2002), 
changes on the BDI-II greater than 8.46 are considered to be a reliable change (i.e., improvement), and if, simultaneously, the score falls below 14.29, the client is considered 'recovered'. Regarding the OQ-45.2 IR subscale, the client is considered dysfunctional when the score is above 14 points. Change is considered reliable (RCI) when a score changes by eight points or more.

Definition of an event. The event should be a binomial variable that occurs after a period, which in this study is defined as recovery and improvement (as described above). A client was only considered improved and recovered if the status was reached and kept until the last session of treatment (Anderson \& Lambert, 2001).

\section{Survival Analysis}

A survival analysis of an event implies that the whole sample has been included in the analysis, i.e., also those participants who did not reach the event of interest during the observation period. The Kaplan-Meier (KM) method (Kaplan \& Meier, 1958), a non-parametric technique for analysing survival data (Harnett et al., 2010; Willett \& Singer, 1993), was used for the present analyses. To evaluate whether or not the KM survival curves were statistically different, the log rank test (Mantel-Cox test) was used.

\section{Effect Sizes}

Pre-treatment to post-treatment ESs using Cohen's $d$ (Cohen, 1988) were calculated for the BDI-II and the OQ45.2 IR subscale. For a more conservative ES estimate of post-treatment, the value of the last observation carried forward (Shao \& Zhong, 2003) was used. Paired $t$-tests were applied to evaluate the statistical significance of pre-treatment to post-treatment differences on the BDI-II and the OQ-45.2 IR subscale.

\section{RESULTS}

One-fourth of the ITT sample $(23.8 \%)$ attended eight sessions or fewer, and almost half of the clients $(49.2 \%)$ attended 16 sessions or more. The mean number of sessions attended was 13.9 sessions $(S D=6.8)$, and the median was 16 . Out of the 63 clients of the total sample, only two $(3 \%)$ had scores in the functional range of the BDI-II, and seven $(11 \%)$ had scores in the functional range of the OQ-45.2 IR subscale at pre-treatment. These clients were also included in all analysis.

\section{Improvement and Recovery on Depressive Symptoms and Interpersonal Problems}

\section{Proportion of Clients Improving}

The survival analysis for depressive symptoms (BDI-II) revealed that $49.2 \%$ of clients improved, whereas only $15.9 \%$ improved in interpersonal problems (OQ-45.2 IR subscale) $\left[x^{2}(1)=15.95, p<0.001\right]$.

\section{Proportion of Clients Recovering}

Similarly to the improvement rates, significantly more clients recovered from their depressive symptoms (34.9\%), when compared with their interpersonal problems $(14.3 \%)$ $\left[x^{2}(1)=7.23, p=0.01\right]$.

\section{Differential Time Effect of Treatment Group}

Separate survival analyses were carried out to assess whether time to improvement and recovery of depressive symptoms and interpersonal problems were associated with treatment assignment. Log rank (Mantel-Cox) tests show that neither for the time to improvement [BDI-II: $x^{2}(1)=0.7, p=0.40$; OQ-45.2 IR subscale: $x^{2}(1)=0.23$, $p=0.63$ ] nor for the time to recovery [BDI-II: $x^{2}(1)=0.06$, $p=0.81$; OQ-45.2 IR subscale: $x^{2}(1)=0.03, p=0.87$ ] the survival curves of the two treatment conditions differ from each other.

Improvement of Depressive versus Interpersonal Problems

As analyses revealed no significant differences for treatment group regarding time to improvement or recovery in depressive symptoms or interpersonal problems, the total sample $(n=63)$ will be used in the following survival analyses, which aim to assess the hypothesis that change in symptoms occurs faster than change in interpersonal problems (Barkham et al., 1996, 2002; Howard et al., 1993; Kopta et al., 1994). Thus, Figures 1 and 2 compare, respectively, the probability rates of improvement and recovery of depressive symptoms and interpersonal problems for the total sample.

Figure 1 shows the survival curves for improvement of depressive symptoms and interpersonal problems. The curves differ significantly from each other $\left[x^{2}(1)=17.12\right.$, $p<0.001]$. Thus, four sessions of psychotherapy are necessary for the $25 \%$ of the sample to improve from depressive symptoms. Comparatively, more than 20 sessions are necessary for the same $25 \%$ of the sample to reach clinical improvement from interpersonal problems. By session 4, only $11.7 \%$ of the sample had improved their interpersonal symptoms.

\section{Recovery of Depressive Symptoms versus Interpersonal Problems}

In Figure 2, the KM survival curves comparing the probability of recovery for depressive symptoms and 


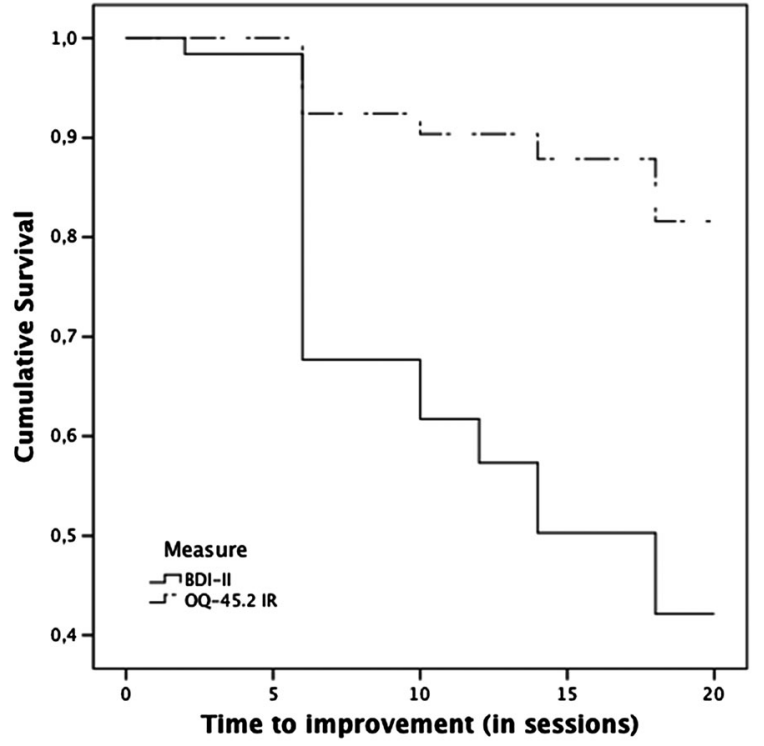

Figure 1. Comparison of Kaplan-Meier survival plots for improving reliably of depressive (Beck Depression Inventory [BDI]-II) and interpersonal problems (Outcome Questionnaire45.2 Interpersonal Relations OQ-45.2 IR subscale) for the entire sample

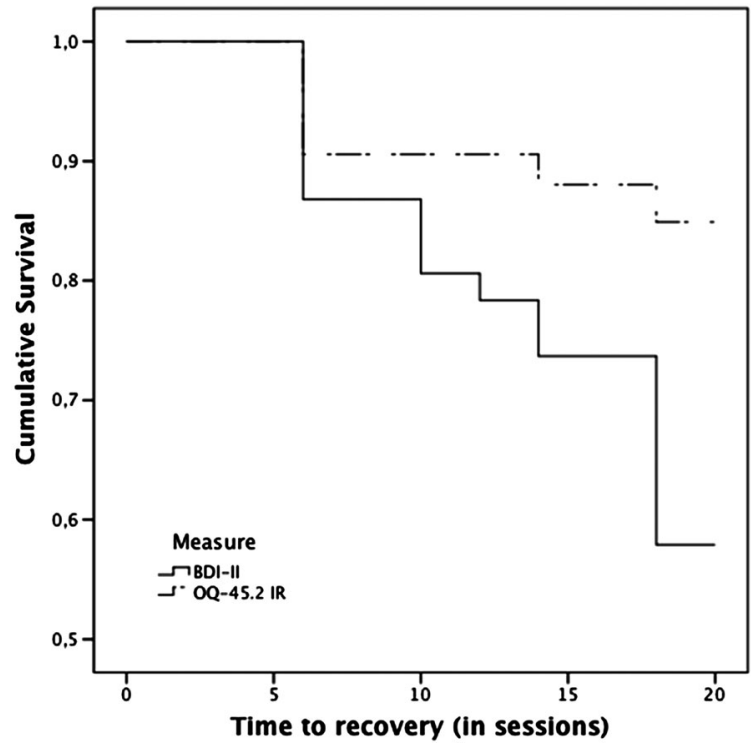

Figure 2. Comparison of Kaplan-Meier survival plots for recovery of depressive (Beck Depression Inventory [BDI]-II) and interpersonal problems (Outcome Questionnaire-45.2 Interpersonal Relations [OQ-45.2 IR] subscale) for the total sample

interpersonal problems are displayed. The survival curves differ marginally from each other $\left[x^{2}(1)=6.65, p=0.10\right]$. They show the same probability of change in the first sessions, but already on the fourth session, the BDI-II curve starts to show a higher probability of improvement per session when compared with the OQ-45.2 IR subscale curve. The differential probability of depressive symptoms and interpersonal problems recovery is clearer by the end of the treatment. At the end of 12 psychotherapy sessions, $25 \%$ of clients had recovered in their depressive symptoms, whereas only $11.5 \%$ had recovered from their interpersonal problems.

Time to Recovery of Depressive Symptoms Only for Those who Recovered

If we considered only clients who recover of depressive symptoms, after four sessions, $25 \%$ of the clients $(n=22)$ had recovered, whereas $50 \%$ recovered only after 11 sessions.

\section{Effect Sizes}

Pre-treatment to post-treatment ESs are an alternative strategy to compare treatment impact on depressive symptoms and interpersonal problems. As Table 1 shows, it was found that the pre-treatment to post-treatment ES value for the BDI-II for the total sample was $d=0.79$, whereas for the OQ-45.2 IR subscale, it was only $d=0.34$. Furthermore, the pre-treatment to post-treatment ESs were statistically significant for the BDI-II but only marginally significant for the OQ-45.2 IR subscale (Table 1).

\section{DISCUSSION}

The main aim of this study was to test whether the time to attain clinically meaningful change was different in NT and CBT. Survival analyses indicate that being assigned to either NT or CBT treatment was not associated with the time to improve or recover. This finding is coherent with the Dodo Bird Verdict (Luborsky, Singer, \& Luborsky, 1975; Luborsky et al., 2006; Stiles, Shapiro, \& Elliott, 1986; Wampold et al., 1997), a paradigm in psychotherapy theory and research that suggests psychotherapy equivalence of all bona fide treatments (Wampold, 2001).

Consistent with the finding that improvement occurs before full recovery (e.g., Anderson \& Lambert, 2001; Kadera et al., 1996), it was found that after four sessions, $25 \%$ of the clients had improved from their depressive symptoms, which is similar to the five sessions for $25 \%$ of improvement reported by Anderson and Lambert (2001). These figures are also very similar to those found by Kadera et al. (1996).

To the authors' knowledge, this is the first research studying the phase model within an NT setting. The fact that a larger proportion of clients improved and recovered from depressive symptoms than from interpersonal 
Table 1. Pre-treatment to post-treatment effect sizes in each outcome measure

\begin{tabular}{|c|c|c|c|c|c|c|c|}
\hline Outcome measure & Treatment group & Pre-treatment mean $(S D)$ & Post-treatment mean $(S D)$ & $d f$ & $t$ & $p$ & $d^{+}$ \\
\hline \multirow[t]{3}{*}{ BDI-II } & CBT & $33.90(11)$ & $18.90(13)$ & 28 & 6.08 & 0.000 & 1.25 \\
\hline & NT & $29.09(10)$ & $22.59(13)$ & 33 & 3.09 & 0.004 & 0.56 \\
\hline & Total & $31.3(10.7)$ & $20.9(13.3)$ & 62 & 6.19 & 0.000 & 0.79 \\
\hline \multirow[t]{3}{*}{ OQ-45.2 IR subscale } & CBT & $21.41(5.8)$ & $18.06(7.6)$ & 28 & 2.37 & 0.025 & 0.50 \\
\hline & NT & $20.70(5.7)$ & $19.15(7.7)$ & 33 & 1.37 & 0.179 & 0.17 \\
\hline & Total & $21.03(5.73)$ & $18.65(7.62)$ & 62 & 2.66 & 0.010 & 0.34 \\
\hline
\end{tabular}

${ }^{\dagger}$ Effect sizes have been categorized along a continuum of 'small' $(0.2=<\mathrm{ES}=<0.5)$, 'medium' $(0.5=<\mathrm{ES}=<0.8)$ and 'large effect' (ES $\left.>=0.8\right)($ Cohen, 1988). $\mathrm{BDI}-\mathrm{II}=$ Beck Depression Inventory-II. OQ-45.2 IR = Outcome Questionnaire Interpersonal Relations. $S D=$ standard deviation. $d f=$ degree of freedom. $d=$ pre-treatment to post-treatment effect size. $\mathrm{CBT}=$ cognitive-behavioural therapy. $\mathrm{NT}=$ narrative therapy.

problems yields evidence for the phase model for NT and CBT of depression. Additionally, the calculation of pretreatment to post-treatment ESs highlights that more consistent change on the BDI-II (medium ES) and only modest change on the OQ-45.2 IR subscale (small ES) occurred in both treatments. Moreover, findings from the survival analyses add the information that not only greater change occurs in the symptomatic level but also that it occurs significantly faster than change in the interpersonal level.

In another study with NT, using a large sample and a standardized treatment procedure, Vromans and Schweitzer (2011) reported similar results. The authors found large pretreatment to post-treatment ES on depressive symptoms (on the BDI-II) and only medium pre-treatment to post-treatment ES on interpersonal problems (on OQ-45.2 IR subscale). This was also observed in a different study with a sample of clients who underwent psychodynamic psychotherapy of depression (Hilsenroth et al., 2001), which may suggest that the phase model might apply to brief psychotherapies for depression in general. However, in order to generalize these findings, further research with other psychotherapy models and settings should be conducted.

\section{Strengths}

The strengths of the study to be highlighted are its innovative nature by showing data from a clinical trial of an understudied form of psychotherapy (NT), its specificity to depressed clients, the use of a survival analytic approach and the use of a standardized evaluation of clinical improvement.

\section{Limitations and Future Directions}

The sample of therapists was composed of a considerable number of post-graduate students. It remains unclear to which extent this may have influenced the results regarding the speed of change. Some evidence suggests that more experienced therapists achieve quicker results than their less experienced colleagues (e.g., Aveline, 2005;
Okiishi, Lambert, Nielsen, \& Ogles, 2003). Alternatively, there are also studies that found no evidence for differential dose-response curves for professionals and therapists in training (e.g., Beutler et al., 2004; Hansen \& Lambert, 2003). In the present study, the clinical experience of the therapist was not associated with the speed of recovery and improvement.

Also, this kind of research may confound the number of psychotherapy sessions one client received with the time elapsed from the first observation to the last. Thus, it should be highlighted that 'the effects could reflect merely the passage of time rather than the dose of treatment' (Barkham et al., 2002, p. 472). This limitation may refer to the area of psychotherapy outcome research in general, but has special relevance for the scientific question raised by this study, namely the time it takes for a client to change.

Future research should assess clients' symptom progress and interpersonal changes at every session during treatment and also evaluate the follow-up period, given the recurrent nature of major depression.

\section{Implications}

The dose-response research has steered the political discussion around the number of psychotherapy sessions offered by health agencies (Anderson \& Lambert, 2001; Hansen et al., 2002; Harnett et al., 2010; Kadera et al., 1996; Lambert, 2007). One of the arguments is that the number of sessions offered by these agencies is often not informed by empirical research and hence set up arbitrarily. As a consequence, it is argued that public health systems and health insurance companies offer less psychotherapy than what would be necessary for clients to attain significant clinical benefits. Our estimates corroborate the dose found by other researchers that between 8 and 21 sessions of psychotherapy are required for $50 \%$ of clients to improve from depressive symptoms. A typical MDD client who recovers does so by session 11.

In that sense, these findings are informative for clinicians to elaborate a more accurate treatment plan and 
$R$. Lopes et al.

adjust clients and therapists' expectations regarding treatment length. It is suggested that clinicians can reduce dropout rate significantly by informing the clients of the expected length of treatment (Swift \& Callahan, 2011). It was also observed that clients who expected to have more psychotherapy sessions in fact end up attending more sessions (Owen, Smith, \& Rodolfa, 2009). Moreover, for the few clients who will not recover during brief interventions, and especially for the clients who present strong interpersonal problems at onset, long-term treatment plans should be considered.

These findings yield additional evidence for the phase model of change in a sample of depressive clients. No empirical support was found for the claim that CBT produces a more 'superficial' symptomatic level (e.g., Dobson \& Dobson, 2009). In NT and even in stronger interpersonally focused treatments (e.g., IPT; Barkham et al., 1996, 2002), changes in interpersonal problems occur but rather slow compared with changes in depressive symptoms. Thus, these findings support the idea shared by clinicians that special interventions should be applied at different phases of treatment, with more emphasis laid on symptomatic relief in the early stages of treatment and focusing on interpersonal issues at later stages (Howard et al., 1993).

\section{ACKNOWLEDGEMENTS}

This article was supported by the Portuguese Foundation for Science and Technology (FCT), by the grant PTDC/ PSI-PCL/121525/2010 (Ambivalence and unsuccessful psychotherapy, 2011-2014) and by the PhD grant [SFRH/ BD/47343/2008]. The authors would like to kindly thank Michael J. Lambert for the valuable input on the data analysis and encouragement. An earlier version of this article was presented at the 43rd International Meeting of the Society for Psychotherapy Research (SPR) held in Virginia Beach, VA, USA, in June 2012.

\section{REFERENCES}

American Psychiatric Association. (1980). DSM-III: Diagnostic and Statistical Manual of Mental Disorders (3rd ed.). Washington, DC.

American Psychiatric Association. (2000). DSM-IV-TR: Diagnostic and Statistical Manual of Mental Disorders (4th Revised ed.). American Psychiatric Press Inc.

Anderson, E. M., \& Lambert, M. J. (2001). A survival analysis of clinically significant change in outpatient psychotherapy. Journal of Clinical Psychology, 57(7), 875-888.

Aveline, M. (2005). The person of the therapist. Psychotherapy Research, 15(3), 155-164. DOI: 10.1080/10503300500090902

Barkham, M., Rees, A., Stiles, W. B., Hardy, G. E., \& Shapiro, D. A. (2002). Dose-effect relations for psychotherapy of mild depression: A quasi-experimental comparison of effects of 2, 8, and
16 sessions. Psychotherapy Research, 12(4), 463-474. DOI: $10.1093 / \mathrm{ptr} / 12.4 .463$

Barkham, M., Rees, A., Stiles, W. B., Shapiro, D. A., Hardy, G. E., \& Reynolds, S. (1996). Dose-effect relations in time-limited psychotherapy for depression. Journal of Consulting and Clinical Psychology, 64(5), 927-935. DOI: 10.1037/0022006X.64.5.927

Bauer, S., Lambert, M. J., \& Nielsen, S. L. (2004). Clinical significance methods: A comparison of statistical techniques. Journal of Personality Assessment, 82(1), 60-70. DOI: 10.1207/ s15327752jpa8201_11

Beck, A. T., Rush, A. J., Shaw, B. F., \& Emery, G. (1979). Cognitive Therapy of Depression. New York: Guilford Press.

Beck, A. T., Steer, R. A., \& Brown, G. K. (1996). Manual for the Beck Depression Inventory-II (BDI-II). San Antonio, TX: Psychological Corporation.

Beck, A. T., Ward, C., Mendelson, M., Mock, J., \& Erbaugh, J. (1961). An inventory for measuring depression. Archives of General Psychiatry, 4, 561-571.

Beutler, L. E., Malik, M., Alimohamed, S., Harwood, T. M., Talebi, H., Noble, S., \& Wong, E. (2004). Therapist variables. In M. J. Lambert (Ed.), Bergin and Garfield's handbook of psychotherapy and behavior change (5th ed, pp. 227-306). New York: Wiley.

Campos, R. C., \& Gonçalves, B. (2011). The Portuguese version of the Beck Depression Inventory-II (BDI-II). European Journal of Psychological Assessment, 27(4), 258-264. DOI: 10.1027/1015$5759 / \mathrm{a} 000072$

Coelho, R., Martins, A., \& Barros, H. (2002). Clinical profiles relating gender and depressive symptoms among adolescents ascertained by the Beck Depression Inventory II. European Psychiatry, 17(4), 222-226. DOI: 10.1016/S0924-9338(02)00663-6

Cohen, J. (1988). Statistical power analysis for the behavioral sciences (2nd ed). Hillsdale, NJ: Erlbaum.

De Jong, K., Nugter, M. A., Polak, M. G., Wagenborg, J. E. A., Spinhoven, P., \& Heiser, W. J. (2007). The outcome questionnaire (OQ-45) in a Dutch population: A cross-cultural validation. Clinical Psychology \& Psychotherapy, 14(4), 288-301. DOI: $10.1002 /$ cpp. 529

Dobson, D., \& Dobson, K. S. (2009). Evidence-Based Practice of Cognitive-Behavioral Therapy. New York/London: Guilford Press.

Fennel, M. J. (1997). Depressão. In K. Hawton, P. M. Salkovskis, J. Kirk, E D. M. Clark.Terapia cognitivo-comportamental para problemas psiquiátricos. Porto Alegre, RS, Brasil: Artmed.

First, M. B., Gibbon, M., Spitzer, R. L., Williams, J., \& Benjamin, L. (1997). Structured Clinical Interview for DSM-IV axis II personality disorders: SCID-II. Washington, DC: American Psychiatric Press Inc.

First, M. B., Spitzer, R., Gibbons, M., \& Williams, J. (2002). Structured Clinical Interview for DSM-IV-TR axis I disorders, research version, non-patient edition (SCID-I/NP). New York, NY: Biometrics Research, New York State Psychiatric Institute.

Gonçalves, M. M., \& Bento, T. (2008). Manual Terapêutico Psicoterapia Narrativa de Re-Autoria. Portugal: Braga.

Hansen, N. B., \& Lambert, M. J. (2003). An evaluation of the doseresponse relationship in naturalistic treatment settings using survival analysis. Mental Health Services Research, 5(1), 1-12.

Hansen, N. B., Lambert, M. J., \& Forman, E. M. (2002). The psychotherapy dose-response effect and its implications for treatment delivery services. Clinical Psychology: Science and Practice, 9(3), 329-343. DOI: 10.1093/clipsy.9.3.329

Harnett, P., O'Donovan, A., \& Lambert, M. J. (2010). The dose response relationship in psychotherapy: Implications for social policy. Clinical Psychologist, 14(2), 39-44. 
Hatchett, G. T., \& Park, H. L. (2003). Comparison of four operational definitions of premature termination. Psychotherapy: Theory, Research, Practice, Training, 40(3), 226-231. DOI: 10.1037/0033-3204.40.3.226

Hatchett, G. T., Han, K., \& Cooker, P. G. (2002). Predicting premature termination from counseling using the butcher treatment planning inventory. Assessment, 9(2), 156-163. DOI: $10.1177 / 10791102009002006$

Hilsenroth, M. J., Ackerman, S. J., \& Blagys, M. D. (2001). Evaluating the phase model of change during short-term psychodynamic psychotherapy. Psychotherapy Research, 11(1), 29-47. DOI: $10.1080 / 713663851$

Horowitz, L. M., Alden, L. E., Wiggins, J. S., \& Pincus, A. L. (2000). IIP, Inventory of Interpersonal Problems Manual. New York, NY: Psychological Corporation.

Horowitz, L. M., Rosenberg, S. E., Baer, B. A., Ureño, G., \& Villaseñor, V. S. (1988). Inventory of interpersonal problems: Psychometric properties and clinical applications. Journal of Consulting and Clinical Psychology, 56(6), 885-892. DOI: 10.1037/0022-006X.56.6.885

Howard, K. I., Kopta, S. M., Krause, M. S., \& Orlinsky, D. E. (1986). The dose-effect relationship in psychotherapy. American Psychologist, 41(2), 159-164. DOI: 10.1037/0003-066X.41.2.159

Howard, K. I., Lueger, R. J., Maling, M. S., \& Martinovich, Z. (1993). A phase model of psychotherapy outcome: Causal mediation of change. Journal of Consulting and Clinical Psychology, 61(4), 678-685. DOI: 10.1037/0022-006X.61.4.678

Jacobson, N. S., \& Truax, P. (1991). Clinical significance: A statistical approach to defining meaningful change in psychotherapy research. Journal of Consulting and Clinical Psychology, 59(1), 12-19.

Kadera, S. W., Lambert, M. J., \& Andrews, A. A. (1996). How much therapy is really enough? A session-by-session analysis of the psychotherapy dose-effect relationship. Journal of Psychotherapy Practice \& Research, 5(2), 132-151.

Kaplan, E. L., \& Meier, P. (1958). Nonparametric estimation from incomplete observations. Journal of the American statistical association, 53, 457-481.

Kopta, S. M., Howard, K. I., Lowry, J. L., \& Beutler, L. E. (1994). Patterns of symptomatic recovery in psychotherapy. Journal of Consulting and Clinical Psychology, 62(5), 1009-1016.

Lambert, M. J. (2007). Presidential address: What we have learned from a decade of research aimed at improving psychotherapy outcome in routine care. Psychotherapy Research, 17(1), 1-14. DOI: $10.1080 / 10503300601032506$

Lambert, M. J., Burlingame, G. M., Umphress, V., Hansen, N. B., Vermeersch, D. A., Clouse, G. C., \& Yanchar, S. C. (1996a). The reliability and validity of the Outcome Questionnaire. Clinical Psychology \& Psychotherapy, 3(4), 249-258.

Lambert, M. J., Hansen, N. B., Umphress, V., Lunnen, K., Okiishi, J., Burlingame, G. M., \& Reisenger, C. W. (1996b). Administration and scoring manual for the Outcome Questionnaire (OQ 45.2). Stevenson, MD: American Professional Credentialing Services LLC.

Leahy, R. L., \& Holland, S. J. (2000). Treatment plans and interventions for depression and anxiety disorders. New York, NY: The Guilford Press.

Lopes, R., Gonçalves, M. M., Machado, P. P., Sinai, D., Bento, T., \& Salgado, J. (2013). Narrative therapy vs. cognitivebehavioral therapy for moderate depression: Empirical evidence from a controlled clinical trial.

Luborsky, L., Rosenthal, R., Diguer, L., Andrusyna, T. P., Berman, J. S., Levitt, J. T., ... Krause, E. D. (2006). The Dodo Bird Verdict is alive and well-mostly. Clinical Psychology: Science and Practice, 9(1), 2-12. DOI: 10.1093/clipsy.9.1.2

Luborsky, L., Singer, B., \& Luborsky, L. (1975). Comparative studies of psychotherapies: Is it true that "everyone has won and all must have prizes"? Archives of General Psychiatry, 32(8), 995.

Ogles, B. M., Lunnen, K. M., \& Bonesteel, K. (2001). Clinical significance: History, application, and current practice. Clinical Psychology Review, 21(3), 421-446. DOI: 10.1016/S0272-7358(99) 00058-6

Okiishi, J., Lambert, M. J., Nielsen, S. L., \& Ogles, B. M. (2003). Waiting for supershrink: An empirical analysis of therapist effects. Clinical Psychology \& Psychotherapy, 10(6), 361-373. DOI: $10.1002 /$ cpp.383

Owen, J., Smith, A., \& Rodolfa, E. (2009). Clients' expected number of counseling sessions, treatment effectiveness, and termination status: using empirical evidence to inform session limit policies. Journal of College Student Psychotherapy, 23(2), 118-134. DOI: $10.1080 / 87568220902743660$

Richmond, R. (1992). Discriminating variables among psychotherapy dropouts from a psychological training clinic. Professional Psychology: Research and Practice, 23(2), 123-130.

Rush, A. J., Beck, A. T., Kovacs, M., \& Hollon, S. D. (1977). Comparative efficacy of cognitive therapy and pharmacotherapy in the treatment of depressed outpatients. Cognitive Therapy and Research, 1(1), 17-37. DOI: 10.1007/BF01173502

Seggar, L. B., Lambert, M. J., \& Hansen, N. B. (2002). Assessing clinical significance: Application to the Beck Depression Inventory. Behavior Therapy, 33(2), 253-269.

Shao, J., \& Zhong, B. (2003). Last observation carry-forward and last observation analysis. Statistics in Medicine, 22(15), 2429-2441. DOI: 10.1002/sim.1519

Stiles, W. B., Shapiro, D. A., \& Elliott, R. (1986). Are all psychotherapies equivalent? American Psychologist, 41(2), 165-180. DOI: 10.1037/0003-066X.41.2.165

Swift, J. K., \& Callahan, J. L. (2011). Decreasing treatment dropout by addressing expectations for treatment length. Psychotherapy Research, 21(2), 193-200. DOI: 10.1080/ 10503307.2010.541294

Swift, J. K., Callahan, J. L., Heath, C. J., Herbert, G. L., \& Levine, J. C. (2010). Applications of the psychotherapy phase model to clinically significant deterioration. Psychotherapy: Theory, Research, Practice, Training, 47(2), 235-248. DOI: 10.1037/a0019787

Vromans, L. P., \& Schweitzer, R. D. (2011). Narrative therapy for adults with major depressive disorder: Improved symptom and interpersonal outcomes. Psychotherapy Research, 21(1), 4-15. DOI: $10.1080 / 10503301003591792$

Wampold, B. E. (2001). The Great Psychotherapy Debate, Second Edition: Models, Methods, and Findings. Mahwah, NJ: Erlbaum.

Wampold, B. E., Mondin, G. W., Moody, M., Stich, F., Benson, K., \& Ahn, H. N. (1997). A meta-analysis of outcome studies comparing bona fide psychotherapies: Empirically, "all must have prizes." Psychological Bulletin, 122(3), 203-215.

White, M. (2007). Maps of narrative practice. New York, NY: WW Norton \& Company.

White, M., \& Epston, D. (1990). Narrative means to therapeutic ends. New York, NY: WW Norton \& Company.

Willett, J. B., \& Singer, J. D. (1993). Investigating onset, cessation, relapse, and recovery: Why you should, and how you can, use discrete-time survival analysis to examine event occurrence. Journal of Consulting and Clinical Psychology, 61(6), 952-965. DOI: $10.1037 / 0022-006 \times .61 .6 .952$ 